\title{
RESISTÊNCIA DE PLANTAS AOS HERBICIDAS INIBIDORES DA ENZIMA ACETIL COENZIMA A CARBOXILASE (ACCase)
}

\author{
Dirceu Agostinetto ${ }^{1}$, Ribas Antônio Vidal ${ }^{2}$, \\ Nilson Gilberto Fleck ${ }^{2}$ e Mauro Antonio Rizzardi ${ }^{3}$
}

\begin{tabular}{l}
${ }^{1}$ Eng ${ }^{\circ}$. Agrônomo, M. Sc. Aluno do Programa de Pós-Graduação em Fitotecnia. Faculdade de Agronomia da UFRGS. Caixa Posta 776. Porto \\
Alegre, RS 91501-970 dirceua@ig.com.br \\
${ }^{2}$ Eng. Agrônomo, Ph.D., Professor. Departamento de Plantas de Lavoura da UFRGS. Bolsista do CNPq. \\
${ }^{3}$ Eng $^{\circ}$. Agrônomo, M. Sc., Aluno do Programa de Pós-Graduação em Fitotecnia. Faculdade de Agronomia da UFRGS. \\
\hline
\end{tabular}

RESUMO

Plantas daninhas resistentes aos herbicidas inibidores da enzima ACCase apresentam grande importância econômica no mundo, devido à área infestada e ao limitado número de herbicidas alternativos e/ou mecanismos de ação disponíveis para seu controle. Esta revisão teve por objetivo elucidar os mecanismos de resistência de plantas daninhas e de tolerância de plantas cultivadas aos herbicidas inibidores da ACCase. A resistência de plantas daninhas aos inibidores de ACCase deve-se a alterações no local de ação, metabolização e repolarização da membrana. A tolerância das culturas a estes herbicidas deve-se a alterações no local de ação e metabolização. O desenvolvimento de cultivares tolerantes aos inibidores de ACCase permitirá o controle seletivo de plantas daninhas, constituindo-se em alternativa promissora para manejar e controlar espécies daninhas atualmente problemáticas. A introdução de herbicidas com diferentes mecanismos de ação para controlar poáceas ou o uso de inibidores de ACCase em conjunto a herbicidas com diferentes mecanismos de ação, associado ao manejo integrado das plantas daninhas, são estratégias importantes a se adotar para evitar o surgimento de resistência e prolongar o período de uso desses herbicidas.

Palavras-chave: plantas daninhas, plantas cultivadas, inibidores de ACCase, mecanismo de ação.

\section{ABSTRACT \\ Plant resistance to herbicides inhibitors of the enzyme acetyl coenzyme A carboxilase (ACCase)}

Weeds resistant to herbicide inhibitors of the enzyme ACCase present great economic importance throughout the world, due to the wide area infested and limited number of alternative herbicides and/or mechanisms of action availables for their control. This literature review intends to elucidate the mechanisms of weed resistance and of crop tolerance to herbicides inhibitors of ACCase. Weed resistance to ACCase inhibitors is due to alterations in the action site, plant metabolism, and membrane repolarization. Crop tolerance to these herbicides is due to alterations in the action site and to metabolism. The development of tolerant cultivars to ACCase inhibitors will allow selective control of weeds, constituting a promising alternative to manage and control weed species currently problematic. The introduction of herbicides with different mechanisms of action for grass weed control or the use of ACCase inhibitors jointly with herbicides differing in mechanism of action, associated with integrated weed management, are important strategies to be adopted in order to avoid appearance of resistance and to extend the period of utilization of such herbicides.

Key words: ACCase inhibitors, crop, mechanism of action, weeds. 


\section{INTRODUÇÃO}

Os herbicidas inibidores da enzima acetil-Coenzima A carboxilase (ACCase; Ec 6.4.1.2) caracterizam-se por inibir reversível e não competitivamente esta enzima, a qual apresenta papel fundamental na síntese de malonil-CoA, bloqueando, desta forma, a reação inicial da rota metabólica da síntese de lipídios (Vidal, 1997). Os lipídios são componentes essenciais da membrana lipoprotéica da célula e de suas organelas. A inibição de sua síntese afeta a constituição das membranas, alterando a permeabilidade ao fluxo de prótons e, consequientemente, a produção de energia (ATP), levando a planta à morte.

Nas últimas duas décadas, os herbicidas inibidores da enzima ACCase têm assumido grande importância, por possibilitarem o controle seletivo em pós-emergência de espécies da família poaceae em culturas dicotiledôneas e, em alguns casos, também em culturas monocotiledôneas (Kuk et al., 2000). Os herbicidas desta classe foram desenvolvidos na década de 70 e poucos anos após serem lançados, ocuparam rapidamente parte significativa do mercado de produtos graminicidas, sendo divididos em dois grupos químicos: ariloxifenoxipropionatos (AFP) e ciclohexanodionas (CHD). De acordo com Heap (1997), os inibidores de ACCase representam aproximadamente $5 \%$ do total de herbicidas comercializados no mundo. Ainda, segundo o autor, plantas daninhas poáceas resistentes aos inibidores de ACCase apresentam grande importância econômica, devido à extensa área infestada no mundo e ao limitado número de herbicidas alternativos e/ou mecanismos de ação disponíveis para seu controle.

O uso continuado dos herbicidas AFP e CHD passou a provocar elevada pressão de seleção, favorecendo o desenvolvimento de biótipos de plantas resistentes. Embora seja difícil prever o período para surgimento de população resistente, estima-se que sejam necessários mais de 4 anos de aplicações seguidas de herbicidas com mesmo mecanismo de ação. Entretanto, em situações onde são realizadas repetidas aplicações em um mesmo ano, este período pode ser reduzido. Para a espécie Digitaria sanguinalis (L.) Scop. foram necessárias seis aplicações de fluazifop-p, em 4 anos seguidos, para surgir resistência (Hidayat \& Preston, 1997). Porém, para populações de Avena fatua L. submetidas à aplicação de outros herbicidas durante vários anos, uma única aplicação de imazamethabenz selecionou plantas sete vezes mais resistentes que as suscetíveis (Friesen et al., 2000).

Atualmente, em todo o mundo existem 25 relatos de espécies daninhas resistentes aos herbicidas inibidores de ACCase em 22 países, representando aproximadamente $12 \%$ do total de casos de resistência (Weed Science, 2001). No Brasil, há relatos de resistência em Brachiaria plantaginea (Link) Hitch. aos herbicidas sethoxydim, fluazifop, haloxyfop e fenoxaprop (Gazziero et al., 2000; Weed Science, 2001). As plantas daninhas resistentes aos inibidores de ACCase que apresentam maior ocorrência no mundo são A. fatua, Lolium rigidum Gaud. e Lolium multiflorum Lam.

O objetivo desta revisão foi evidenciar os mecanismos de resistência de plantas daninhas e tolerância de plantas cultivadas aos herbicidas inibidores da enzima ACCase.

\section{Tipos de resistência e fatores que favorecem a sua ocorrência}

A resistência de plantas daninhas aos herbicidas é definida como a habilidade herdada pelo biótipo em sobreviver à aplicação herbicida para a qual a população original era suscetível (Vidal, 1997). Na literatura, várias são as referências de ocorrência de biótipos com resistência cruzada aos herbicidas inibidores de ACCase. Este tipo de resistência ocorre quando apenas um mecanismo de ação confere resistência do biótipo a dois ou mais herbicidas. Neste sentido, Gelmini et al. (2000) verificaram que um biótipo de B. plantaginea resistente aos inibidores de ACCase apresentou diferentes níveis de resistência cruzada aos herbicidas fluazifop e sethoxydim. Também Cortez et al. (2000) analisaram a sensibilidade de plantas inteiras a testes "in vitro" com a enzima extraída de três biótipos resistentes e um suscetível, coletados em diferentes locais, tendo verificado que as doses-resposta obtidas pela aplicação dos herbicidas sethoxydim, fluazifop e fenoxaprop foram diferentes entre os biótipos e várias vezes superiores em relação ao biótipo suscetível, Isso levou os autores a concluir que a resistência era cruzada e a diversidade dos padrões de resistência encontrados, bem como a coevolução da resistência, em vários locais, podem ser considerados como fatores que, possivelmente, dificultarão o manejo da resistência.

Para resistência múltipla, que ocorre quando dois ou mais mecanismos distintos conferem resistência aos herbicidas, poucos casos são relatados na literatura. A espécie L. rigidum, encontrada na Austrália, destaca-se por apresentar resistência múltipla a herbicidas com sete mecanismos de ação diferentes (Weed Science, 2001). Segundo Powles \& Preston (1998), biótipos de L. rigidum apresentam maior nível de resistência aos herbicidas AFP em relação aos CHD. De acordo com Christoffoleti et al. (2001), as plantas de B. plantaginea resistente aos herbicidas inibidores de ACCase não apresentam resistência múltipla aos produtos de manejo com mecanismos de ação alternativos (glyphosate, paraquat, glufosinate, sulfosate, paraquat+diuron e MSMA). Desse modo, esses herbicidas podem ser utilizados no período de entre-safra para manejo de populações resistentes em áreas que utilizam o sistema de semeadura direta.

De acordo com Vidal (1997), fatores importantes, em relação às características das plantas daninhas, dos herbicidas e das práticas culturais, devem ser considerados para se evitar rápida ocorrência de resistência a determinado herbicida. Dentre as características ligadas às plantas daninhas, citamse: hábito de crescimento anual, elevada capacidade de produção de sementes, alta percentagem de sementes viáveis 
(baixa dormência), produção de várias gerações a cada ano e sensibilidade a determinado herbicida. As características relacionadas aos herbicidas incluem: atuação em único local de ação, elevada eficiência sobre determinada espécie daninha (causando alta pressão de seleção), longa atividade residual no solo, uso de doses superiores às necessárias para alcançar controle das plantas daninhas e utilização freqüente em aplicações seqüênciais. Dentre as práticas culturais, destacamse: prática de monocultivo, não eliminação das plantas daninhas que escapam ao controle por herbicidas e uso contínuo de um único ou de poucos herbicidas que apresentam o mesmo modo de ação.

\section{Caracterização da ACCase e dos locais de ação dos inibidores na enzima}

A enzima ACCase catalisa a conversão de acetilcoenzima A (acetil-CoA) em malonil-coenzima A (malonilCoA), através da adição de CO à acetil-CoA (Hess, 1997). Essa conversão é o primeiro pašso na biossíntese de lipídios e pode determinar a velocidade da rota metabólica. A enzima ACCase caracteriza-se por apresentar três centros de reação: a enzima biotina carboxilase, a proteína carreadora de biotina (PCB) e a enzima carboxitransferase. A ACCase extraída, purificada e parcialmente caracterizada em milho, demonstrou que a enzima também utiliza propionil-CoA carboxilase como substrato, embora a eficiência desse seja cerca de $50 \%$ menor do que o substrato acetil-CoA (Egli et al., 1993).

Em estudos de dupla ligação, verificou-se que os herbicidas AFP e CHD podem ligar-se na mesma região da ACCase, pois se excluem reciprocamente em uma região comum de ligação (Redina et al., 1990). No entanto, Burton et al. (1991) mostraram evidências de que tal ligação ocorra em diferentes sublocais dentro do local de ação, sugerindo que o local de ligação, embora aparente ser único, apresenta superposição de sublocais para ligação desses herbicidas. Por sua vez, Taylor et al. (1995) propuseram duas classes de inibidores de ACCase compartimentalizados numa porção hidrofóbica comum em pequena região acil do substrato acetilCoA, sugerindo a formação de conjugados de AFP e CHD, o que pode servir como multilocal inibidor que potencializaria a ação dos mesmos. Os autores verificaram que ésteres de AFP conjugados com CoA mostraram-se 150 vezes mais potentes em inibir ACCase em trigo do que as formas ácidas.

Segundo Hess (1997), todos os trabalhos que testaram a sensibilidade da ACCase obtida de espécies dicotiledôneas, demonstraram que os herbicidas AFP e CHD apresentam baixa ou nenhuma redução na atividade da enzima e que o mecanismo de ação para os herbicidas dessas classes é a inibição da enzima ACCase. Além disso, o mecanismo de seletividade entre dicotiledôneas e monocotiledôneas é o local de ação da ACCase, não tendo sido referidas diferenças significativas na absorção, translocação ou metabolismo entre as espécies dessas classes.

\section{Resistência das plantas dicotiledôneas vs. suscetibilidade das monocotiledôneas}

Resultados de pesquisas demonstram existir dois tipos de enzima ACCase nas plantas: uma forma eucariótica (tipo I), constituída por três grupos funcionais em uma única proteína com peso molecular de aproximadamente $230 \mathrm{kDa}$ e outra forma procariótica (tipo II), composta por três subunidades de proteínas [biotina carboxilase (49 kDa); proteína transportadora de biotina $(17 \mathrm{kDa})$; e, carboxitransferase, com duas subunidades, $\alpha$ (32 kDa) e $\beta(80 \mathrm{kDa})]$ (Li \& Conan, 1992; Alban et al., 1994). A ACCase na forma eucariótica é inibida pelos herbicidas AFP e CHD, enquanto a forma procariótica da enzima não é afetada.

A tolerância de espécies dicotiledôneas aos herbicidas inibidores da ACCase deve-se à enzima presente no cloroplasto ser do tipo procariótica, enquanto a localizada no citoplasma é eucariótica. Trabalho de pesquisa com plantas dicotiledôneas demonstrou que a inibição da forma eucariótica do citoplasma não afetou a biossíntese de ácidos graxos com 16 ou 18 átomos de carbono, uma vez que as reações de síntese destes compostos ocorrem somente no cloroplasto (Konishi \& Sasaki, 1994).

Espécies da família Poaceae caracterizam-se por apresentarem a forma eucariótica da enzima tanto no citosol como no plastídio, o que as tornam sensíveis aos herbicidas inibidores de ACCase (Sasaki et al., 1995). Especula-se que durante a evolução das gramíneas, a adição de uma seqüência de DNA, codificando um domínio no plastídio para a forma eucariótica de ACCase, tenha provocado a retirada do gene da subunidade $\beta$ do complexo transcarboxilase do genoma do plastídio e, provavelmente, também de genes de outras subunidades de ACCase do genoma nuclear (Sasaki et al., 1995). De acordo com Konishi \& Sasaki (1994), nessas plantas a forma procariótica foi perdida devido à ausência de gene accD, responsável pela codificação da enzima carboxitransferase nos cloroplastos.

\section{MECANISMOS DE RESISTÊNCIA DE PLANTAS DANINHAS}

As diferentes mutações ocorridas no gene que codifica a enzima ACCase e o tipo de alelo deste gene podem ser causas de diferenças no nível de resistência. Pesquisas demonstram não existir variações na absorção e/ou translocação de herbicidas AFP e CHD entre biótipos resistentes e suscetíveis (Maneechote et al., 1997; Hidayat \& Preston, 1997; Kuk 
Dirceu Agostinetto et al.

et al., 1999). Entretanto, análise da ACCase de vários biótipos resistentes sugere a presença de diferentes mutações, resultando em padrão variável de resistência aos herbicidas AFP e CHD (Devine, 1997). Ainda, segundo o autor, em todos os casos analisados a resistência aos inibidores da ACCase é codificada por um gene nuclear dominante ou semidominante.

A resistência de plantas daninhas aos inibidores de ACCase pode ser influenciada pelos seguintes fatores: mudanças no local de ação, metabolização e detoxificação e repolarização das membranas.

\section{a) Mudanças no local de ação}

Alterações no local de ação, tornando a ACCase insensível, é o mecanismo mais comum de resistência aos herbicidas AFP e CHD (Seefeldt et al., 1996; Sankula et al., 1997; Kuk et al., 1999; Kuk et al., 2000) (Figura 1). Este mecanismo normalmente é demonstrado por extração e purificação da enzima ACCase a partir de tecidos de folhas jovens, seguido pela quantificação da atividade enzimática em presença de herbicidas (Cortez et al., 2000). Foi constatado que a enzima ACCase, oriunda de biótipos resistentes ao diclofop exibiu sensibilidade 50 vezes menor quando comparada com a do

\section{b) Metabolização}

O aumento da degradação do herbicida em determinadas plantas pode conferir resistência aos herbicidas inibidores de ACCase. Biótipos de L. rigidum e Alopecurus myosuroides Huds., resistentes a compostos deste grupo de herbicidas, exibem elevada expressão ou atividade das enzimas citocromo $\mathrm{P}$ monooxigenase, as quais comumente estão envolvidas no processo inicial do metabolismo herbicida em plantas (Devine, 1997). Ainda, segundo o autor, dependendo da formade citocromo $\mathrm{P}$ envolvido, este mecanismo também pode conferir resistência para outras classes de herbicidas com diferentes mecanismos de ação.

Em biótipos de Avena esterilis L., o incremento do metabolismo aumentou em 1,5 vezes a detoxificação de diclofop, comparativamente ao biótipo suscetível, demonstrando que o biótipo resistente é mais hábil em converter diclofop em compostos não tóxicos (Maneechote et al., 1997). De igual modo, a atividade da enzima ACCase não foi alterada por aplicação de fluazifop e a taxa de metabolismo do herbicida foi aproximadamente 1,5 vezes mais rápida no biótipo resistente de $D$. sanguinalis, quando comparada ao biótipo suscetível, o que

Figura 1: Mecanismo proposto de alteração da enzima ACCase e que confere resistência aos herbicidas inibidores de ACCAse.

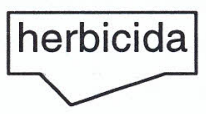

Biótipo suscetível

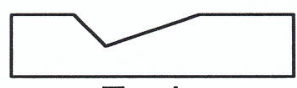

Enzima
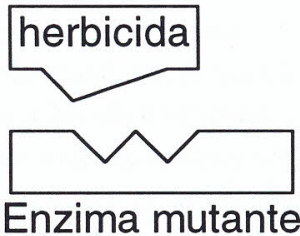

Biótipo resistente

Enzima mutante

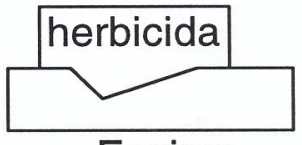

Enzima

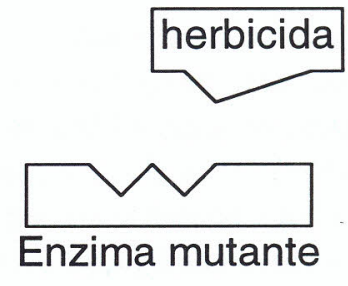

biótipo suscetível, o que o tornou seis vezes mais resistente (Maneechote et al. 1997). De modo similar, a enzima ACCase de biótipo resistente de Lolium temulentum L. foi 833 e 10 vezes mais resistente ao diclofop e sethoxydim, respectivamente, que o biótipo suscetível (Kuk et al., 2000). Em biótipo de Setaria faberi Herrm., foi verificado que a elevada resistência ao sethoxydim estava relacionada à reduzida sensibilidade da ACCase (Sankula et al., 1997). Contudo, os estudos realizados até o momento não permitem afirmar se todos os biótipos resistentes apresentam a mesma mutação da enzima ACCase, embora a variabilidade na resistência para diferentes inibidores de ACCase sugira que possam estar envolvidas diferentes mutações na ACCase. conferiu resistência 20 vezes maior para o biótipo resistente (Hidayat \& Preston, 1997).

A constatação de resistência aos herbicidas de mesmo ou de diferente mecanismo de ação, para a mesma espécie vegetal, sugere que mais de um mecanismo de resistência possa estar envolvido (Heap, 1997). Neste contexto, Powles \& Preston (2000) observaram que populações de Hordeum leporinum Link, resistentes aos inibidores de ACCase, metabolizaram 1,3 vezes mais fluazifop do que as plantas suscetíveis. Além disso, tanto plantas resistentes quanto suscetíveis apresentaram igual taxa de metabolização de sethoxydim, o que segundo os autores, demonstra que o mecanismo de resistência ao fluazifop e a outros AFP deve-se à detoxificação do herbicida, enquanto para sethoxydim o mecanismo de re- 
sistência, aparentemente, é a modificação do local de ação. Esses resultados, bem como os obtidos por Hidayat \& Preston (1997), demonstram que a metabolização pode ser o mecanismo responsável pela ocorrência de resistência a apenas um herbicida. Por outro lado, a ocorrência de resistência múltipla em Echinochloa phylopogon (Stapf) Koss pode derivar de um único mecanismo de resistência, qual seja, o aumento da degradação do herbicida (Fischer et al., 2000). No entanto, as evidências dos dados não demonstram que o metabolismo seja um mecanismo relevante de resistência de plantas daninhas aos herbicidas AFP e CHD (Seefeldt et al., 1996; Kuk et al., 1999).

\section{c) Repolarização das membranas}

A manutenção da polaridade das membranas é fundamental para a sobrevivência da célula vegetal. Assim, plantas resistentes aos herbicidas inibidores de ACCase devem possuir mais de um mecanismo que proporcione resistência, um que esteja relacionado com a ACCase e outro com a membrana plasmática. Em biótipos de L. rigidum, a repolarização das membranas ocorre independentemente da presença da ACCase mutada, sendo constatadas várias diferenças entre as membranas dos biótipos resistentes e sensíveis (Heap, 1997). Assim os biótipos resistentes de L. rigidum e A. fatua não apresentaram ACCase insensível ou metabolismo da molécula, mas evidenciaram diferenças no efeito dos herbicidas sobre as funções da membrana plasmática.

O herbicida diclofop despolariza rapidamente a membrana dos biótipos resistentes e sensíveis; contudo, os biótipos resistentes são capazes de reverter o processo rapidamente (Devine, 1997). A aplicação de diclofop em biótipos resistente e suscetível de $A$. sterilis causou despolarização de membranas. Porém, ambos os biótipos foram hábeis em repolarizar as membranas, demonstrando que mudanças nas propriedades da mesma não contribuem para a resistência (Maneechote et al., 1997). Análise da composição lipídica da membrana plasmática, visando determinar a base do efeito diferencial de diclofop no potencial elétrico transmembrana, entre biótipos resistentes e suscetíveis de $A$. fatua, demonstrou que as diferenças entre biótipos no transporte de diclofop pela membrana e o potencial da membrana plasmática não estão relacionadas às diferenças em sua composição lipídica (Renault et al., 1997). A eficácia do mecanismo de repolarização das membranas está diretamente ligada à capacidade das plantas em remover rapidamente as moléculas herbicidas do meio, ligando-as às células da parede celular ou acumulando-as nos vacúolos.

\section{TOLERÂNCIA DE PLANTAS CULTIVADAS}

A seletividade das culturas aos herbicidas inibidores de ACCase deve-se à metabolização do composto ou à insensibilidade da enzima. A metabolização dos compostos AFP, após ativados por enzimas carboxiesterases que rompem as ligações éster e originam parentes ácidos desses compostos, dá-se através de hidroxilação, sendo o grupo hidroxila rapidamente conjugado com glicose. De acordo com Mcfadden et al. (1989), a metabolização por hidroxilação de diclofop em trigo é catalisada por uma enzima citocromo $\mathrm{P}$ monooxigenase, embora o metabolismo de fenoxaprop em ${ }_{450}^{450}$ e cevada possa ocorrer por conjugação com glutationa (Tall et al., 1995). De forma semelhante à metabolização de diclofop em trigo, também espécies daninhas, como A. fatua, podem transformar o herbicida em um composto glicosil-éster não tóxico; porém, a reação é reversível, de forma que o herbicida torna-se tóxico para a espécie (Holtum et al., 1991).

O desenvolvimento de cultivares resistentes aos inibidores de ACCase tem proporcionado outra opção para o manejo e controle de plantas daninhas em culturas onde estes herbicidas não poderiam ser utilizados. A resistência aos AFP e CHD foi selecionada por exposição de células aos inibidores de ACCase. A base para a resistência foi, na maioria das vezes, uma mutação no local de ação da enzima ACCase (Devine \& Shimabukuro, 1994). Porém, diferentes mutações do local da enzima ACCase resultaram em padrões variáveis de resistência aos herbicidas AFP e CHD (Beckie et al., 2000). Alterações na enzima ACCase de milho tornaram-no resistente a aplicações de até $840 \mathrm{~g} / \mathrm{ha}$ de sethoxydim (Vangessel et al., 1997). A isoenzima ACCase isolada de milho resistente apresentou 3,7, 77 e 12,8 vezes maior resistência do que a do milho suscetível para clethodim, sethoxydim e talkoxydim, respectivamente (Incledon \& Hall, 1999). Adicionalmente, Gengenbach et al. (1999) verificaram que plântulas de milho, selecionadas em culturas de tecidos, aumentaram a atividade da enzima ACCase em 100 e 16 vezes para sethoxydim e haloxyfop, respectivamente, em comparação ao tipo silvestre, levando os autores a concluirem que seja este o mecanismo de tolerância aos herbicidas inibidores de ACCase.

O controle de plantas espontâneas de milho resistente aos herbicidas inibidores de ACCase tem-se mostrado problemático para implantação da rotação de culturas, especialmente quando esta envolve a soja. Neste sentido, trabalhos de pesquisa têm avaliado os níveis de resistência cruzada do milho resistente aos demais herbicidas AFP e CHD. Desse modo, Young \& Hart (1997) observaram, em trabalhos de casa de vegetação e em condições de campo, que o milho mutante é menos resistente ao clethodim, quando comparado aos demais herbicidas inibidores de ACCase. Da mesma forma, Vangessel et al. (1997) verificaram que clethodim, quando aplicado sobre híbridos de milho resistentes ao sethoxydim, nas doses de 140 e $560 \mathrm{~g} / \mathrm{ha}$, causou dano de 85 e $99 \%$, respectivamente. Isso os levaram a concluir que clethodim pode ser usado para controlar plantas espontâneas de milho que são resistentes ao sethoxydim. 


\section{CONSIDERAÇÕES FINAIS}

Variações no mecanismo de resistência entre espécies de plantas daninhas e a possibilidade de ocorrência de mais de um mecanismo na mesma espécie, aumentam a probabilidade de ocorrência de resistência cruzada ou múltipla aos herbicidas inibidores de ACCase. Esse fato, associado ao uso continuado desses compostos, reduzem o período hábil de utilização agrícola desses produtos.

O principal mecanismo de resistência de plantas daninhas aos herbicidas inibidores de ACCase é a insensibilidade da enzima. Porém, a metabolização, aliada à aplicação de subdoses ou a condições pouco favoráveis, pode favorecer o surgimento de resistência a herbicida específico, aumentando a ocorrência de biótipos resistentes.

$\mathrm{O}$ desenvolvimento de cultivares tolerantes aos herbicidas inibidores de ACCase permitirá o controle seletivo de plantas daninhas em culturas, constituindo-se em alternativa promissora para manejar e controlar espécies atualmente problemáticas.

A introdução de herbicidas com diferentes mecanismos de ação para o controle de poáceas ou o uso de herbicidas inibidores de ACCase, em conjunto com herbicidas de diferentes mecanismos de ação, associados ao manejo integrado das plantas daninhas, são estratégias importantes a serem adotadas para evitar o surgimento de resistência e prolongar o período de uso desses herbicidas.

\section{LITERATURA CITADA}

ALBAN, C.; BALDET, P.; DOUCE, R. Localization and characterization of two structurally different forms of acetyl-CoA carboxylase in young pea leaves, of which one is sensitive to aryloxyphenoxypropionate herbicides. Biochemical Journal, v.300, n.2, p.557-565, 1994.

BECKIE, H.J.; HEAP, I.M.; SMEDA, R.J.; HALL, L.M. Screening for herbicide resistance in weeds. Weed Technology, v.14, n.2, p.428-445, 2000.

BURTON, J.D.; GRONWALD, J.W.; KEITH, D.A.; SOMERS, D.A.; GENGENBACH, B.G.; WYSE, D.L. Kinetics of inhibition of acetyl-coenzime A carboxylase by sethoxydim and haloxyfop. Pesticide Biochemistry and Physiology, n.39, v.1, p.100-109, 1991.

CHRISTOFFOLETI, P.J.; KEHDI, C.A.; CORTEZ, M.G. Manejo da planta daninha Brachiaria plantaginea resistente aos herbicidas inibidores da ACCase. Planta Daninha, v.19, n.1, p.61-66, 2001.

CORTEZ, M.G.; CHRISTOFFOLETI, P.J.; VICTÓRIA FILHO, R.; PRADO, R.do. Resistência cruzada e mecanismo de resistência em biótipos de Brachiaria plantaginea resistentes a herbicidas inibidores de ACCase. In: CON-
GRESSO BRASILEIRO DA CIÊNCIA DAS PLANTAS DANINHAS, 22. Foz do Iguaçu, 2000. Resumos... Londrina: SBCPD, 2000. p.498.

DEVINE, M.D. Mechanisms of resistance to acetyl-coenzyme A carboxylase inhibitors: a review. Pesticide Science, v.51, n.2, p. 259-264, 1997.

DEVINE, M.D.; SHIMABUKURO, R.H. Resistance to acetyl coenzyme A carboxylase inhibiting herbicides. In: POWLES, S. B.; HOLTUM, J. A. M., eds. Herbicide resistance in plants: biology and biochemistry. Boca Raton: CRC Press, 1994. p.141-169.

EGLI, M.A.; GENGENBACH, B.G.; GRONWALD, J.W.; SOMERS, D.A.; WYSE, D.L. Characterization of maize acetyl-coenzyme A carboxylase. Plant Physiology, v.101, n.2, p.499-506, 1993.

FISCHER, A.J.; ATEH, C.M.; BAYER, D.E.; HILL, J.E. Herbicide-resistant Echinochloa oryzoides and $E$. phyllopogon in California Oryza sativa fields. Weed Science, v.48, n.2, p.225-230, 2000.

FRIESEN, L.F.; JONES, T.L.; VAN ACKER, R.C.; MORRISON, I.N. Identification of Avena fatua populations resistant to imazamethabenz, flamprop, and fenoxaprop-p. Weed Science, v.48, n.5, p.532-540, 2000.

GAZZIERO, D.L.P.; CHRISTOFFOLETI, P.J.; BRIGHENTI, A.M.; PRETE, C.E.C.; VOLL, E. Resistência da planta daninha capim-marmelada (Brachiaria plantaginea) aos herbicidas inibidores da enzima ACCase na cultura da soja. Planta Daninha, v.18, n.1, p.169-180, 2000.

GELMINI, G.A.; VICTÓRIA FILHO, R.; ADORYAN, M.L.; NOVO, M.C.S. Resistência de Brachiaria plantaginea aos herbicidas inibidores da ACCase. In: CONGRESSO BRASILEIRO DA CIÊNCIA DAS PLANTAS DANINHAS, 22. Foz do Iguaçu, 2000. Resumos... Londrina: SBCPD, 2000. p.499.

GENGENBACH, B.G.; VANDEE, K.L.; EGLI, M.A.; HILDEBRANDT, K.M.; YUN, S.J.; LUTZ, S.M.; MARSHALL, L.C.; WYSE, D.L.; SOMERS, D.A. Genetic relationships of alleles for tolerance to sethoxydim herbicide in maize. Crop Science, v.39, n.3, p.812-818, 1999.

HEAP, I.M. The occurrence of herbicides-resistant weeds worldwide. Pesticide Science, v.51, n.3, p.235-243, 1997.

HESS, F.D. Mode of action of lipid biosynthesis inhibitors (graminicides - ACCase inhibitors). In: Herbicide Action Course, 1997. West Lafayette: Purdue University, 1997. p.209-309. 
HIDAYAT, I.; PRESTON, C. Enhanced metabolism of fluazifop acid in a biotype of Digitaria sanguinalis resistant to the herbicide fluazifop-p-butyl. Pesticide Biochemistry and Physiology, v.57, n.2, p.137-146, 1997.

HOLTUM, J.A.M.; MATTHEWS, J.M., HÄUSLER, E.R.; POWLES, S.B. Cross resistance to herbicides in annual ryegrass (Lolium rigidum). III: On the mechanism of resistance to diclofop-methyl. Plant Physiology, v.97, n.3, p.1026-1034, 1991.

INCLEDON, B.J.; HALL, J.C. Inhibition of ACCase220 and ACCase 240 isozymes from sethoxydim-resistant and susceptible maize hybrids. Journal of Agricultural and Food Chemistry, v.47, n.1, p.229-304, 1999.

KONISHI, T; SASAKI, Y. Compartmentalization of two forms of acetyl-CoA carboxylase in plants and the origin of their tolerance toward herbicides. Proceedings of the National Academy of Sciences of the United States of America,v.91, n.9, p. 3598-3601, 1994.

KUK, Y.I.; BURGOS, N.R.; TALBERT, R.E. Cross- and multiple resistance of diclofop-resistant Lolium spp. Weed Science, v.48, n.4, p.412-419, 2000.

KUK, Y.I; WU, J.; DERR, J.F; HATZIOS, K.K. Mechanism de fenoxaprop resistance in an accession of smooth crabgrass (Digitaria ischaemum). Pesticide Biochemistry and Physiology, v.64, n.2, p.112-123, 1999.

LI, S.J.; CONAN JR., J.E. The genes encoding the two carboxytransferase subunits of Escherichia coli acetyl-CoA carboxylase. Journal Biology Chemical, v.267, n.24, 16841-16847, 1992.

MANEECHOTE, C.; PRESTON, C.; POWLES, S.B. A diclofopmethyl-resistance Avena sterilis biotype with a herbicideresistant acetyl-coenzyme A carboxylase and enhanced metabolism of diclofop-methyl. Pesticide Science, v.49, n.1, p.105-114, 1997.

MCFADDEN, J.J.; FREAR, D.S.; MANSAGER, E.R. Aryl hydroxylation of diclofop by a cytrochrome P-450 dependent monooxygenase from wheat. Pesticide Biochemistry and Physiology, v.34, n.1, p. 92-100, 1989.

POWLES, S.B.; PRESTON, C. Herbicide cross resistance and multiple resistance in plants, 1998. Disponível na Internet. http://www.ncsu.edu/org/hrac/momo2 em 27 mai. 1998.

POWLES, S.B.; PRESTON, C. Mechanisms of resistance to acetyl-coenzyme A carboxylase-inhibiting herbicides in a Hordeum leporinum population. Pest Management Science, v.56, n.5, p.441-447, 2000.
REDINA, A.R.; CRAIG-KENNARD, A.C.; BEAUDOIN, J.D.; BREEN, M.K. Inhibition of acetyl-coenzyme A carboxylase by two classes of grass-selective herbicides. Journal Agricultural Food Chemistry, v.38, n.4, p.1282$1287,1990$.

RENAULT, S.; SHUKLA, A.E.; GIBLIN, M.; MACKENZIE, S.L.; DEVINE, M.D. Plasma membrane lipid composition and herbicide effects on lipoxygenase activity do not contribute to differential membrane responses in herbicide-resistant and -susceptible wild oat (Avena fatua L.) biotypes. Journal of Agricultural and Food Chemistry, v.45, n.8, p.3269-3275, 1997.

SANKULA, A.; DUPONT, S.; DEVINE, M.D. Resistance to ACCase-inhibitor herbicides in wild oat: evidence for target site-based resistance in two biotypes from Canada. Pesticide Biochemistry and Physiology, v.57, n.1, p.147$155,1997$.

SASAKI, Y.; KONISHI, T.; NAGANO, Y. The compartmentation of acetyl-coenzyme A carboxylase in plants. Plant Physiology, v.108, n.2, p.445-449, 1995.

SEEFELDT. S.S.; FUERST, E.P.; GEALY, D.R.; SHUKLA, G.P.I.; DEVINE, M.D. Mechanisms of resistance to diclofop of two wild oat (Avena fatua) biotypes from the Willamette Valley of Oregon. Weed Science, v.44, n.4, p.776-781, 1996.

TAL, J.A.; HALL, J.C. STEPHENSON, G.R. Non-enzymatic conjugation of fenoxaprop-etyl with glutathione and cysteine in several grass species. Weed Research, v.35, n.3, p.133-139, 1995.

TAYLOR, W.S.; HIXON, M.; CHI, H.; MARSILII, E., RENDINA, A.R. Inhibition of acetyl-coenzyme A carboxylase by coenzyme A conjugates of grassselective herbicides. Pesticide Science, v.43, n.2, p.177180, 1995.

VANGESSEL, M.J.; JOHNSON, Q.; ISAACS, M. Response of sethoxydim-resistant corn (Zea mays) hybrids to postemergence graminicides. Weed Technology, v.11, n.3, p.598-601, 1997.

VIDAL, R.A. Herbicidas: mecanismos de ação e resistência de plantas. Porto Alegre: Ribas Vidal, 1997. 165p.

WEED SCIENCE. Herbicide resistant weeds by country, 2001. Disponível na Internet. http://weedscience.org em 06 mai. 2001.

YOUNG, B.G.; HART, S.E. Control of volunteer sethoxydimresistant corn (Zea mays) in soybean (Glycine max). Weed Technology, v.11, n.4, p.649-655, 1997. 